\title{
AUTOMATED DETECTION OF PAROXYSMAL GAMMA WAVES IN MEDITATION EEG
}

\author{
Manuel A.Vázquez ${ }^{a *}$, Jing Jin ${ }^{b \dagger}$, Justin Dauwels ${ }^{b}$ François B. Vialatte ${ }^{c}$ \\ ${ }^{a}$ Universidad Carlos III de Madrid, Depto. de Teoría de la Señal y Comunicaciones, Madrid, Spain 28903 \\ ${ }^{b}$ Nanyang Technological University, School of Electrical and Electronic Engineering, Singapore 639798 \\ ${ }^{c}$ ESPCI ParisTech, Laboratoire SIGMA, 75231 Paris Cedex 05, France
}

\begin{abstract}
Meditation is a fascinating topic, yet has received limited attention in the neuroscience and signal processing community so far. A few studies have investigated electroencephalograms (EEG) recorded during meditation. Strong EEG activity has been observed in the left temporal lobe of meditators. Meditators exhibit more paroxysmal gamma waves (PGWs) in active regions of the brain. In this paper, a method is proposed to automatically detect PGWs from meditation EEG. The proposed algorithm is able to identify multiple sources in the brain that generate PGWs, and the sources associated with different types of PGWs can be distinguished. The effectiveness of the proposed method is assessed on 3 subjects possessing different degrees of expertise in practicing a yoga type meditation known as Bhramari Pranayama.
\end{abstract}

Index Terms - Paroxysmal gamma wave; Electroencephalogram; Meditation; Bhramari Pranayama; Spike detection

\section{INTRODUCTION}

Meditation refers to a family of self regulation practices focusing on training attention and awareness, so as to bring mental processes under greater voluntary control. However, despite almost 50 years of study, the psychophysiological properties and personality traits of meditation have not been adequately studied $[1,2]$. There are various types of meditation techniques, such as transcendental meditation, Buddhist meditation, and yoga type meditation. In this paper, we consider EEG recorded during Bhramari Pranayama (BhPr), which is a yoga type meditation where periods of long exhalation and short inhalation are alternated. BhPr may help to ease up the hormonal imbalance manifestation, such as hypertension, anxiety, and abnormal blood pressure [3].

Neuroscientists have investigated brain activity during meditation. Significantly enhanced activation has been ob-

\footnotetext{
${ }^{*} \mathrm{M}$. V. acknowledges the support of the Ministry of Education of Spain (Programa Nacional de Movilidad de Recursos Humanos del Plan Nacional de I-D+i 2008-2011).

$\dagger$ J. J. and M. V. equally contributed to this paper.
}

served in the temporal lobe during meditation in several fMRI studies $[4,5]$. EEG studies on meditation have revealed enhanced alpha band activity during meditation $[6,7,8]$. An increase of activity in the gamma band of the EEG during meditation has also been reported $[2,9,10]$; in particular, subjects exhibit more paroxysmal gamma waves (PGWs). The knowledge of the PGW distribution may help us to have a better understanding of the brain activity during meditation. However, comprehensive PGW marking is time consuming, which motivates us to develop automated PGW detection algorithms.

In this paper, we propose a fully automated procedure for extracting and clustering PGWs in EEG recordings of meditators, by combining several signal processing techniques including principal component analysis (PCA), blind signal separation (BSS), and K-means clustering. The proposed procedure is able to identify multiple sources that generate PGWs, and associate sources with different types of PGWs. In contrast, only a single source was identified in prior work [2], by manually screening the PGWs.

This paper is organized as follows: in Section 2, we explain our proposed PGW detection method; in Section 3, we present and discuss the results, and in Section 4, we draw conclusions about our findings.

\section{METHODS}

\subsection{Bhramari Pranayama EEG Recording}

We consider 3 different subjects practicing BhPr meditation: There is a beginner " $B$ " without training, an intermediate subject " $I$ " trained for a month, and an expert " $E$ " trained for 4 months. EEG was recorded in one or two consecutive BhPr sessions in which each subject performed approximately 20 breathing episodes. Each episode in turn consists of a short inhalation period and a long exhalation period, during which the subject produces a typical humming sound. An ambient sound signal was recorded along with the EEG to reliably indicate the periods of exhalation or inhalation. EEG was also recorded before and after BhPr in the resting condition. The EEG recordings were conducted in an electrically shielded room using a Biosemi system with 128 active electrodes. The 


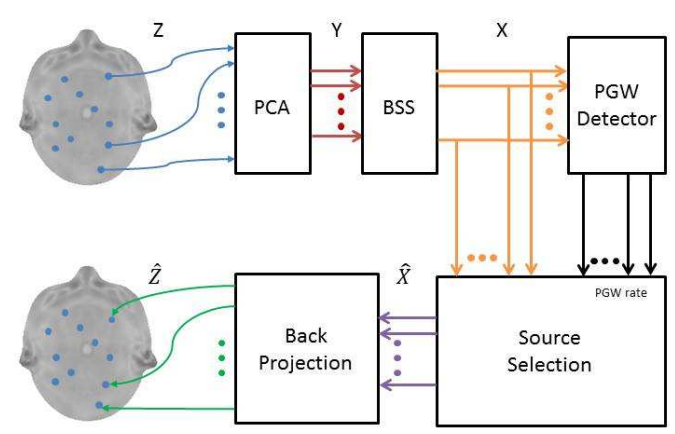

Fig. 1. Diagram of the proposed procedure.

signals were sampled at $2048 \mathrm{~Hz}$ and an analog filter between 1 and $300 \mathrm{~Hz}$ was applied. Moreover, notch filters were applied at $50 \mathrm{~Hz}$ and every harmonic of $25 \mathrm{~Hz}$ to remove the power line interferences.

\subsection{Signal Processing}

A diagram of the proposed procedure is depicted in Fig. 1. Principal component analysis (PCA) [11] is the first step in the processing flow, so as to compress the 128-channel EEG recording into data with a smaller number of channels. Next, blind signal separation (BSS) [12] is applied to identify the sources from the compressed EEG. We estimate the PGW rate for each source, and select the sources that generate most of the PGWs; we backproject the latter in sensor domain, to reconstruct the 128-channel EEG recording. In the following, we describe the different steps of the proposed signal processing procedure.

\subsubsection{Principal Component Analysis}

Principal component analysis (PCA) [11] is a common technique for finding patterns in high-dimensional data. We apply PCA to reduce the number of EEG channels. Let $Z \in \mathbb{R}^{N \times M}$ denote the $N$-channel EEG recordings, where $M$ is the number of samples in each time series. The importance of each eigenvector of the covariance matrix $R$ of $Z$ can be quantitatively measured by its associated eigenvalue: the larger the eigenvalue, the more relevant the corresponding eigenvector will be in explaining the data. Denoting the eigenvalues of $R$ by $\lambda_{1}, \lambda_{2}, \cdots, \lambda_{N}$ in descending order and the corresponding eigenvectors by $u_{1}, u_{2}, \cdots, u_{N}, Z$ can be compressed by retaining the top $L$ (with $L<<N$ ) dimensions, most relevant to interpret the data. The compressed data $Y \in \mathbb{R}^{L \times M}$ can be obtained as:

$$
Y=\left[\begin{array}{llll}
u_{1} & u_{2} & \cdots & u_{L}
\end{array}\right]^{T} Z .
$$

The parameter $L$ is chosen such that

$$
\frac{\sum_{i=1}^{L} \lambda_{i}}{\sum_{i=1}^{N} \lambda_{i}} \geq \gamma,
$$

with $\gamma \in(0,1]$ (we choose $\gamma=0.95$ ).

\subsubsection{Blind Signal Separation}

Blind signal separation (BSS) [12] is a well-known technique to reconstruct unobserved sources from multi-sensor recordings. The adjective "blind" stresses the fact that the sources are not observed, and no information is available about the mixture [12]. In our setting, we assume that the compressed data $Y$ (1), resulting from PCA, is a linear combination of independent sources $X \in \mathbb{R}^{L \times M}$ located in the brain:

$$
Y=A X+N,
$$

where $A \in \mathbb{R}^{L \times L}$ is the mixing matrix and $N \in \mathbb{R}^{L \times M}$ is i.i.d. Gaussian noise. We aim at reconstructing $X$ from $Y$ :

$$
X=W Y
$$

where $W \in \mathbb{R}^{L \times L}$ is unknown. We apply the BSS method "Second Order Blind Identification" (SOBI) [13] to infer the weight matrix $W$ :

$$
\hat{W}=\hat{U}^{H}\left[\left(\lambda_{1}-\hat{\sigma}^{2}\right)^{-0.5} u_{1}, \cdots,\left(\lambda_{L}-\hat{\sigma}^{2}\right)^{-0.5} u_{L}\right]^{H},
$$

where $\hat{\sigma}^{2}$ represents the estimated noise variance, computed using resting EEG. The unitary matrix $\hat{U}$ is obtained as joint diagonalizer of the sample estimates.

\subsubsection{Paroxysmal Gamma Wave Detection}

The paroxysmal gamma waves (PGWs) in meditation EEG are characterized by distinct high-frequency biphasic patterns, with a shape close to a neural spike [2] (see Fig. 2). The large amplitude voltage transients occur at sustained fast rhythms of $10-30 \mathrm{~Hz}$. Manually extracting PGWs is time consuming, which motivated us to develop automated procedures to extract PGWs. In this paper, we extract PGWs from various signals: from the sources obtained through BSS (cf. Section 2.2.2), from the backprojected signals in sensor domain (see Fig. 4b-e), and from the raw EEG (see Fig. 4a).

Our PGW detection method exploits morphological aspects of PGWs, as illustrated in Fig. 2. The following conditions are evaluated by our proposed method: the peak value $V_{p 1}$ of the main lobe (first peak) is supposed to be larger than a several standard deviation of the signal, i.e., $V_{p 1}>\alpha \sigma_{x}$, where $\sigma_{x}$ is the standard deviation of the entire signal and $\alpha$ is a scalar; the minor peak (second peak) $V_{p 2}$ is supposed to be significantly smaller than the main peak $V_{p 1}$, i.e. $\rho_{1} V_{p p 1} \leq$ $V_{p p 2} \leq \rho_{2} V_{p p 1}$ with $\rho_{1}<\rho_{2}<1$; the trough should be below the onset point, i.e. $V_{o n}>V_{t r}$; and finally, the duration $T$ of the PGW should be smaller than a constant $\tau$.

The proposed method is "adaptive", catering to each individual channel (or source), as the parameters are learnt from $K$ PGW samples manually extracted from each individual channel (or source). In our analysis, we set $K=60$ for each channel (or source). The ratio of number of training samples to testing samples is maintained at $\sim 1: 5$. 
For each individual PGW $k$ (with $k=1,2, \cdots, K$ ) at each channel (or source) $i$, we compute the parameters such as $\alpha(i, k), \rho_{1}(i, k), \rho_{2}(i, k)$ and $\tau(i, k)$ (see Fig. 2). We determine the extreme values, i.e. $\alpha_{i}=\min _{k} \alpha(i, k), \rho_{1, i}=$ $\min _{k} \rho_{1}(i, k), \rho_{2, i}=\max _{k} \rho_{2}(i, k)$ and $\tau_{i}=\max _{k} \tau(i, k)$, as the final representatives for channel (or source) $i$. With this choice of parameters, the automated morphological detector considers all manually selected PGWs as positives, as they meet the selection conditions. Next, with this set of parameters for each individual channel (or source), we apply the detector to all channels (or sources), in order to extract the PGWs. The proposed method is computationally efficient, which is important for real-time EEG processing and off processing of large EEG data sets.

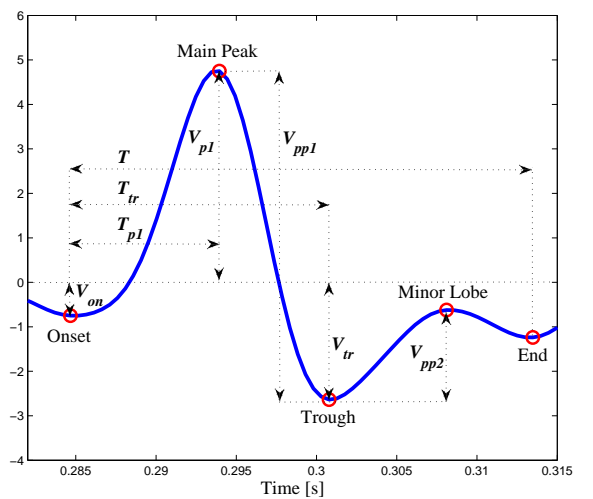

Fig. 2. Morphological parameters of a PGW.

\subsubsection{Clustering}

Once all PGWs have been extracted at each source, we apply an unsupervised classifier, specifically K-means clustering [14], allowing us to distinguish different PGW shapes. Three features are used for clustering: timing of the main peak $T_{p 1}$, timing of the main trough $T_{t r}$, and the slope of the rising flank of the main peak.

\subsubsection{PGW Sources and Back Projection}

After obtaining the PGWs for all sources, we identify the sources that contain the most PGWs. Concretely, we discard sources with a total number of PGWs below a certain percentile $Q_{\lambda}$. Also sources with PGW rate below a certain threshold rate $v$ are removed. In our numerical analysis, we set $Q_{\lambda}=80 \%$ and $v=10 \mathrm{~Hz}$ (as in [2]). We then back project the remaining sources, with high PGW rate and largest number of PGWs, on the scalp [15], similarly as in (3). We use the MATLAB toolbox EEGLAB [16] to visualize the reconstructed signals onto a 2-D top-view head model.

\section{RESULTS AND DISCUSSION}

\subsection{Sensitivity and Specificity Analysis}

We conducted a sensitivity and specificity analysis of the proposed automated PGW detector. Several experts manually ex- tracted PGWs from a 18 s long exhalation period of EEG from subject $B$; we applied the PGW detector on the same EEG segment, in addition to the single-threshold method [17] and the "double threshold with filtering" method [18]. In Fig. 3, the results are summarized. On the left hand side, a $0.4 \mathrm{~s}$ snapshot of the EEG segment is shown, with the outcomes of the three detectors and the manually labeled PGWs; on the right hand side, the receiver operating characteristic (ROC) curves for all 3 methods are shown. Clearly, the proposed morphological PGW detector outperforms both the singlethreshold method [17] and the "double threshold with filtering" method [18].
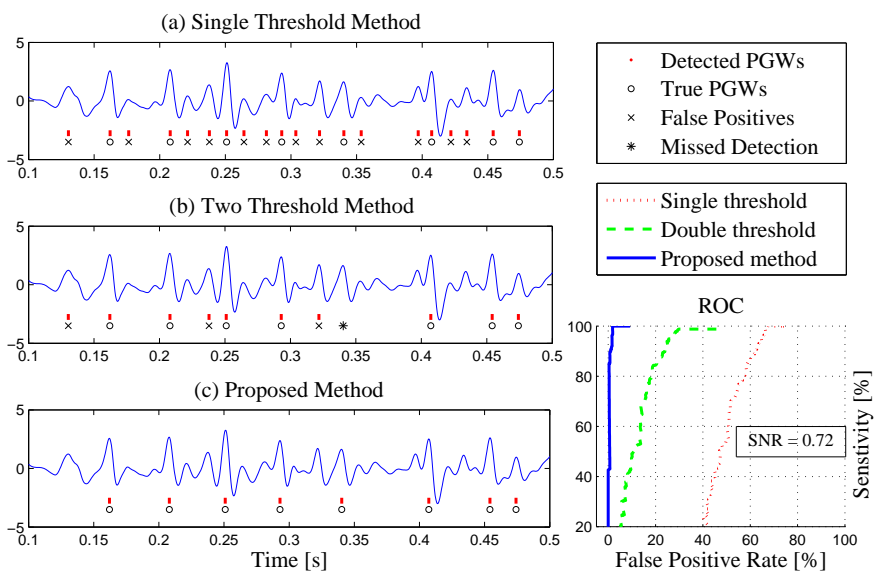

Fig. 3. Comparison of PGW detection results (left), and ROC curves (right).

As can be seen also from Table 1 (with threshold =1.0), the proposed method has the lowest false positive (FP) rate, and the highest sensitivity and specificity; although it involves more PGW features, it has a comparably small processing time (for a single PGW).

Table 1. Comparison of PGW detection methods FP Rate Sensitivity Specificity Features Processing Time

$\begin{array}{lccccc}\text { Single-threshold } & 67.6 \% & 100 \% & 49.6 \% & 1 & 12.0 \mathrm{~ms} \\ \text { Filtered 2-threshold } & 29.8 \% & 98.8 \% & 80.0 \% & 3 & 41.9 \mathrm{~ms} \\ \text { Proposed method } & 2.0 \% & 100 \% & 99.1 \% & 4 & 34.6 \mathrm{~ms}\end{array}$

\subsection{Single Source}

A prior study [2] of the same EEG data only considered a single source, manually extracted all the PGWs. A single source was extracted for each episode, without distinguishing the short inhalation and long exhalation period; in other words, the same source was assumed to generate PGWs in both periods, which is a restrictive assumption. In order to compare the proposed method with the results of [2], we first extracted a single source as well (see Table 2 for input settings (bold)). The results are displayed in Fig. 4b; there is a good match between our results to those in [2], as similar regions of the brain seem to be activated during BhPr. For all 
the subjects, the left temporal lobe was active with high PGW rate, which is in agreement with the hypothesis in $[4,5]$ suggesting that meditation is associated with an activation of the left temporal lobe. As comparison, the distribution of PGWs extracted directly from the raw EEG is shown in Fig. 4a.

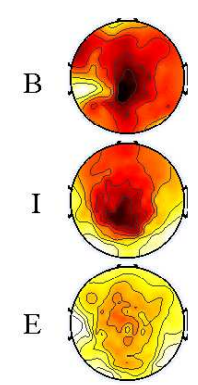

(a)

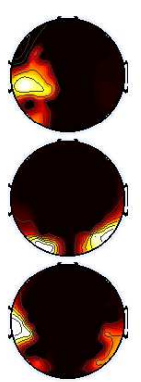

(b)

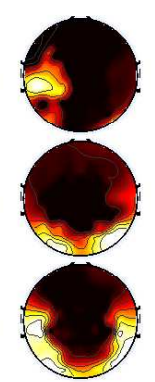

(c) (d)

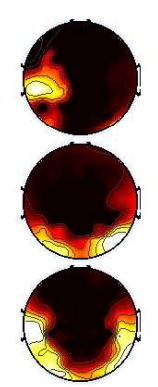

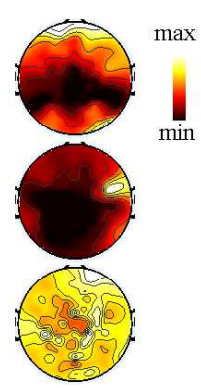

(e)
Fig. 4. PGW rate distribution $[\mathrm{Hz}]$ for (a) raw signal and backprojection from (b) a single source, (c) multiple sources assumption, (d) PGWs from cluster 1 only, and (e) PGWs from cluster 2 only.

Table 2. Parameters of PGW detector in source selection.

\begin{tabular}{c|cc|cccc|ccccccccc} 
& $\boldsymbol{S} \mathbf{1}_{\boldsymbol{B}}$ & $S 2_{B}$ & $\boldsymbol{S} \mathbf{1}_{\boldsymbol{I}}$ & $S 2_{I}$ & $S 3_{I}$ & $S 4_{I}$ & $\boldsymbol{S} \mathbf{1}_{\boldsymbol{E}}$ & $S 2_{E}$ & $S 3_{E}$ & $S 4_{E}$ & $S 5_{E}$ & $S 6_{E}$ & $S 7_{E}$ & $S 8_{E}$ & $S 9_{E}$ \\
\hline$\alpha$ & $\mathbf{1 . 0 2}$ & 1.39 & $\mathbf{1 . 2 0}$ & 1.25 & 1.27 & 1.13 & $\mathbf{1 . 2 0}$ & 1.25 & 1.14 & 1.10 & 1.21 & 1.34 & 1.16 & 1.21 & 1.25 \\
$\rho_{1}\left[10^{-2}\right]$ & $\mathbf{6 . 1}$ & 5.4 & $\mathbf{1 0 . 2}$ & 6.1 & 8.9 & 6.6 & $\mathbf{5 . 6}$ & 5.9 & 6.7 & 5.4 & 8.7 & 6.1 & 6.9 & 5.6 & 5.2 \\
$\rho_{2}\left[10^{-2}\right]$ & $\mathbf{8 4 . 8}$ & 84.9 & $\mathbf{8 4 . 8}$ & 84.8 & 84.9 & 84.8 & $\mathbf{8 4 . 9}$ & 84.8 & 84.9 & 84.9 & 85 & 84.5 & 84.8 & 84.9 & 84.8 \\
$\tau[\mathrm{ms}]$ & $\mathbf{4 8}$ & 46 & $\mathbf{4 3}$ & 51 & 48 & 45 & $\mathbf{5 0}$ & 47 & 46 & 46 & 46 & 47 & 46 & 44 & 45
\end{tabular}

\subsection{Multiple Sources}

Now we consider multiple sources, as described in Section 2.2.5. Results are summarized in Fig. 4c for each subject during one of the exhalation periods (see Table 2 for parameter settings). For subject $B, 2$ sources were selected out of 10 principal components; for $I$, 4 sources were selected out of 47 principal components, and for $E, 9$ sources were selected out of 23 principal components. Interestingly, the additional sources activate mostly the left temporal lobe, similarly as in the single-source analysis. As illustrated in Fig. 5, the PGW distribution during exhalation periods is quite stable, but visibly less stable during inhalation periods.

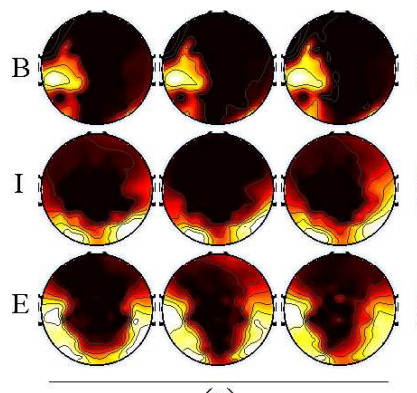

(a)

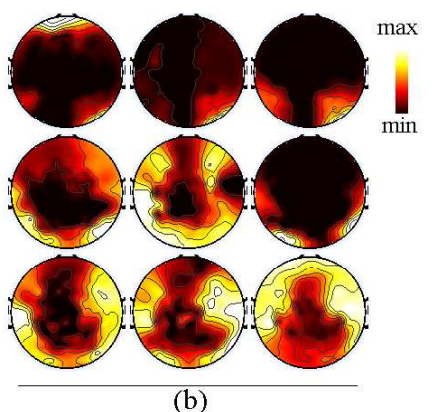

(b)
Fig. 5. PGW rate distribution $[\mathrm{Hz}]$ with multiple-source assumption for backprojection from (a) 3 successive exhalation periods, and (b) 3 successive inhalation periods.

\subsection{Multiple Sources with Clustering}

We applied K-means clustering to the PGWs from multiple sources, as described in Section 2.2.4. For the sake of simplicity, we chose 2 clusters, although the analysis could be straightforwardly repeated with multiple clusters. In Fig. 6, we show PGWs belonging to different clusters, corresponding to different types of PGWs. Comparing to cluster 1, PGWs from cluster 2 have late, smooth and less steep main peaks.

Results for both PGW clusters are shown in Fig. 4d \& e.

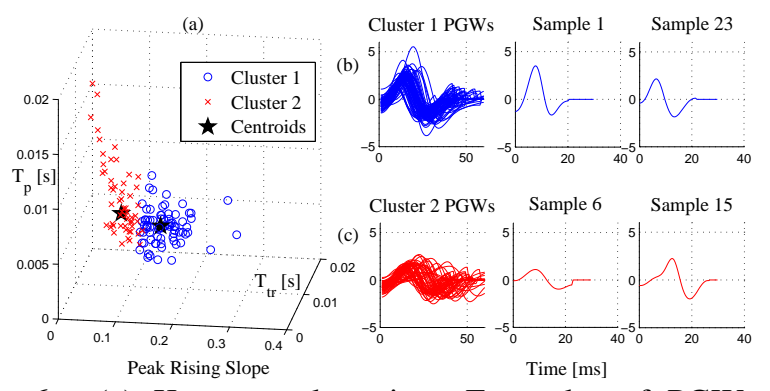

Fig. 6. (a) K-means clustering; Examples of PGWs in (b) Cluster 1 and (c) Cluster 2.

The brain regions associated to the 2 clusters are mostly nonoverlapping, suggesting that different regions of the brain might generate different types of PGWs during meditation. Cluster 1 has a dominant PGW rate at $\sim 10-20 \mathrm{~Hz}$, while it is $<5 \mathrm{~Hz}$ for cluster 2 , which may explain why the PGW distribution associated with cluster 1 (cf. Fig. 4d) is very similar to the PGW distribution associated to multiple sources without clustering (cf. Fig. 4c).

\section{CONCLUSIONS}

We have proposed a fully automated procedure to extract PGWs in EEG of Bhramari Pranayama (BhPr) practitioners. Compared to two other PGW detectors, the proposed method has the lowest false positive rate, highest sensitivity and specificity, and a reasonable processing time. We investigated the effectiveness of the proposed method on the EEG of 3 subjects possessing different degrees of expertise in practicing BhPr. We backprojected EEG from both single and multiple sources of PGWs. The resulting PGW scalp distribution agree well with finding in prior studies $[2,9,10]$. In addition, the proposed method is able to identify multiple PGWs sources in an automated fashion, whereas in prior work [2], a single PGW source was extracted manually, which is a time-consuming process. We also conducted analysis of exhalation periods and inhalation periods separately. For each subject, the PGW distribution during exhalation periods is stable, while it is far less consistent during inhalation periods. We identified 2 different types of PGWs, associated with different brain regions. In future work, we will investigate the synchrony of PGWs across different brain regions, and will extend the study to a larger number of subjects. 


\section{REFERENCES}

[1] T. Takahashi, T. Murata, T. Hamada, M. Omori, H. Kosaka, M. Kikuchi, H. Yoshida, and Y. Wada, "Changes in eeg and autonomic nervous activity during meditation and their association with personality traits," International Journal of Psychophysiology, vol. 55, no. 2, pp. 199-207, 2005.

[2] F. Vialatte, H. Bakardjian, R. Prasad, and A. Cichocki, "Electroencephalographic paroxysmal gamma waves during bhramari pranayama: A yoga breathing technique," Consciousness and cognition, vol. 18, no. 4, pp. 977-988, 2009.

[3] S. Pandey, N. Mahato, and R. Navale, "Role of selfinduced sound therapy: Bhramari pranayama in tinnitus," Audiological Medicine, vol. 8, no. 3, pp. 137-141, 2010.

[4] S. Lazar, G. Bush, R. Gollub, G. Fricchione, G. Khalsa, and $\mathrm{H}$. Benson, "Functional brain mapping of the relaxation response and meditation," Neuroreport, vol. 11, no. 7 , pp. 1581-1585, 2000.

[5] B. Hölzel, U. Ott, H. Hempel, A. Hackl, K. Wolf, R. Stark, and D. Vaitl, "Differential engagement of anterior cingulate and adjacent medial frontal cortex in adept meditators and non-meditators," Neuroscience letters, vol. 421, no. 1, pp. 16-21, 2007.

[6] B. Cahn and J. Polich, "Meditation states and traits: Eeg, erp, and neuroimaging studies.," Psychological bulletin, vol. 132, no. 2, p. 180, 2006.

[7] J. Banquet, "Spectral analysis of the eeg in meditation," Electroencephalography and clinical neurophysiology, vol. 35, no. 2, pp. 143-151, 1973.

[8] F. Travis and R. Wallace, "Autonomic and eeg patterns during eyes-closed rest and transcendental meditation (tm) practice: the basis for a neural model of tm practice," Consciousness and cognition, vol. 8, no. 3, pp. 302-318, 1999.

[9] D. Lehmann, P. Faber, P. Achermann, D. Jeanmonod, L. Gianotti, and D. Pizzagalli, "Brain sources of eeg gamma frequency during volitionally meditationinduced, altered states of consciousness, and experience of the self," Psychiatry Research: Neuroimaging, vol. 108, no. 2, pp. 111-121, 2001.

[10] A. Lutz, L. Greischar, N. Rawlings, M. Ricard, and R. Davidson, "Long-term meditators self-induce highamplitude gamma synchrony during mental practice," Proceedings of the National Academy of Sciences of the United States of America, vol. 101, no. 46, pp. 1636916373, 2004.
[11] L. Smith, "A tutorial on principal components analysis," Cornell University, USA, vol. 51, p. 52, 2002.

[12] J. Cardoso, "Blind signal separation: statistical principles," Proceedings of the IEEE, vol. 86, no. 10, pp. 2009-2025, 1998.

[13] A. Belouchrani, K. Abed-Meraim, J. Cardoso, and E. Moulines, "A blind source separation technique using second-order statistics," Signal Processing, IEEE Transactions on, vol. 45, no. 2, pp. 434-444, 1997.

[14] V. Faber, "Clustering and the continuous k-means algorithm," Los Alamos Science, vol. 22, pp. 138-144, 1994.

[15] J. Karhunen, A. Hyvarinen, R. Vigario, J. Hurri, and E. Oja, "Applications of neural blind separation to signal and image processing," in Acoustics, Speech, and Signal Processing, ICASSP, IEEE International Conference on, vol. 1, pp. 131-134, IEEE, 1997.

[16] A. Delorme and S. Makeig, "Eeglab: an open source toolbox for analysis of single-trial eeg dynamics including independent component analysis," Journal of neuroscience methods, vol. 134, no. 1, pp. 9-21, 2004.

[17] S. Gozani and J. Miller, "Optimal discrimination and classification of neuronal action potential waveforms from multiunit, multichannel recordings using softwarebased linear filters," Biomedical Engineering, IEEE Transactions on, vol. 41, no. 4, pp. 358-372, 1994.

[18] T. Borghi, R. Gusmeroli, A. Spinelli, and G. Baranauskas, "A simple method for efficient spike detection in multiunit recordings," Journal of neuroscience methods, vol. 163, no. 1, pp. 176-180, 2007. 